\title{
In a sphere of their own!
}

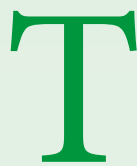

okuyama are technological pioneers in the field of light-cured sub-microscopic spherical filler particles, which has resulted

in composite restorations of outstanding aesthetics, reliability and user-friendliness.

They are the only composite manufacturer to use patented spherical filler particles within their materials. Each variant utilising spherical particles of different diameters to maximise their optical and physical properties for the desired indication.

In addition to optimised optical properties resulting in enhanced aesthetic restorations, Tokuyama's spherical filler particles offer other significant advantages compared with the irregular shaped filler particles used by all other manufacturers. They are quicker and easier to pack into nooks and crannies, reducing the risk of voids; easier to sculpt and carve; and have a much smoother surface finish which has a natural high lustre that requires minimal if any polishing.

Tokuyama's unique spherical filler particles produce the highest gloss in the shortest possible time. Their fillers are not easy to dislodge and create a very smooth surface that is highly resistant to abrasion. Their initial lustre lasts and lasts.

Consequently, Tokuyama spherical fillers ensure smooth restorations that stay smooth; diffuse and transmit light for optimal shading and aesthetics; produce mirror reflection and have high abrasion resistance that ensures a long-lasting polish, lustre, durability and colour stability; and optimum optical shading.

Spherical fillers - A pearl of an idea Utilising patented Sol-Gel Technology Tokuyama 'grow' their spherical filler particles to a diameter that is optimised for their desired colour adaptation and outstanding physical properties.

All other manufacturers simply grind their glass materials until the individual filler particles are within a desired, but random, size range. Their filler particles are all irregular in size and shape, as seen under a scanning electron microscope.

$\rightarrow$ SEM of Tokuyama spherical filler particles

\section{$\rightarrow$ SEM of irregular filler composite material}

What does this mean for the clinician and patient?

\section{Mirror reflection and}

lustre

With their identically shaped spherical filler particles, Tokuyama composites reflect light just like natural enamel and have a natural lustre. This is unlike rough surfaced composites which scatter light diffusely to produce a dull matt appearance.

\section{Abrasion resistance}

Tokuyama composites' uniform and small spherical filler particles are not easy to dislodge and produce a very smooth surface that is highly resistant to abrasion. This abrasion resistant surface remains smooth permanently, so that the initial lustre of Tokuyama composites remains permanent too.

Irregularly shaped filler particles, used by other manufacturers, can become dislodged or plucked out by polishing etc to leave a rough and irregular pitted surface which is very abrasive and very difficult to polish.

\section{Light diffusion and transmission}

The high light diffusion and transmission properties of Tokuyama composites ensure a uniform and gradual transition between tooth and composite. Unlike conventional composites which exhibit minimal light diffusion and transition resulting in visible margins.

\section{Faster polishing}

Tokuyama composites produce the highest gloss in the shortest time.

\section{Radical Amplified Polymerisation (RAP) Technology}

Tokuyama's patented Radical Amplified Polymerisation (RAP) Technology enables them to reuse the camphorquinone over and over again. This not only speeds up the curing time, but also dramatically reduces the amount of camphorquinone that is necessary in other composites.
Longer working time, faster cure Tokuyama’s RAP Technology ensures faster curing plus high resistance to ambient light to ensure an extremely generous working time, when required. Followed by an extremely short curing time. Consequently, Tokuyama composites offer ease of placement, sculpting and finishing which remains completely under your control until the moment you want it to cure, at which point it cures virtually instantaneously.

\section{Deep and completeness of cure}

Tokuyama's RAP Technology ensures a much higher degree of polymerisation resulting in much lower residual monomer, which leads to stronger, deeper and more complete curing.

\section{Minimal shade change}

When camphorquinone is light cured its shade changes to a more yellowish hue.

Tokuyama's RAP Technology means there is a much lower proportion of camphorquinone in their composite materials which ensures an imperceptible shade change after light curing. Unlike other composite brands.

Tokuyama's combination of patented spherical filler particles and RAP Technology means that their composites are easier to place, sculpt and finish; produce smooth restorations which are easier to polish and have a mirror finish and lustre that lasts and lasts and lasts; diffuse and transmit light for optical shading and aesthetics; are extremely abrasion resistant; have optimum optical properties; extended working times; shorter curing times, imperceptible colour change after curing; and are extremely aesthetic. Consequently, Tokuyama composites are in a sphere of their own!

For more information about the complete Tokuyama range contact your local Trycare Representative on 01274885544 or visit www.trycare.co.uk. 